\title{
An Ion Beam Tracking System based on a Parallel Plate Avalanche Counter
}

\author{
I. P. Carter ${ }^{1, a}$, K. Ramachandran ${ }^{1, b}$, M. Dasgupta ${ }^{1}$, D. J. Hinde ${ }^{1}$, R. Rafiei ${ }^{1, c}$, D. H. Luong ${ }^{1}$, E. Williams ${ }^{1}$, K. J. Cook ${ }^{1}$, \\ S. McNeil ${ }^{1}$, D. C. Rafferty ${ }^{1}$, A. B. Harding ${ }^{1}$, A. G. Muirhead ${ }^{1}$, and T. Tunningley ${ }^{1}$. \\ ${ }^{1}$ Department of Nuclear Physics, Research School of Physics and Engineering, Australian National University, ACT 0200, Aus- \\ tralia.
}

\begin{abstract}
A pair of twin position-sensitive parallel plate avalanche counters have been developed at the Australian National University as a tracking system to aid in the further rejection of unwanted beam particles from a $6.5 \mathrm{~T}$ super conducting solenoid separator named SOLEROO. Their function is to track and identify each beam particle passing through the detectors on an event-by-event basis. In-beam studies have been completed and the detectors are in successful operation, demonstrating the tracking capability. A high efficiency 512-pixel wide-angle silicon detector array will then be integrated with the tracking system for nuclear reactions studies of radioactive ions.
\end{abstract}

\section{Radioactive Ion Beams}

Most of our current understanding in nuclear physics has come from using stable targets and beams. Stable nuclei represent only a small fraction of the total number of nuclei that can exist and do not exhibit many of the exotic properties seen in nuclei far from stability, such as halos [1]. The ability to study unstable nuclei has opened up a vast new range of physics to be explored. Whilst decay of these isotopes makes most of them unsuitable as targets, they can be used as beams, known as Radioactive (or Rare) Isotope Beams (RIB) [2]. The Australian National University (ANU) RIB capability, based on a super-conducting solenoidal separator, is currently being developed [3]. This capability is successfully producing radioactive nuclei such as $\left({ }^{6} \mathrm{He},{ }^{8} \mathrm{Li},{ }^{10} \mathrm{Be},{ }^{12} \mathrm{~B}\right)$ by transfer reactions, which are then transmitted and focussed by the solenoid onto the secondary target position for nuclear reaction studies.

\section{SOLEROO}

The RIB capability is based on a $6.5 \mathrm{~T}$ superconducting solenoidal separator SOLEROO (SOLenoidal Exotic Rare isOtOpe separator) shown in Figure 1. This single solenoid separator can achieve RIBs of reasonable purities, but not more than $60 \%$ [4]. Higher beam purities have been achieved at other RIB facilities (e.g. TwinSol [5] and RIBRAS [6]), with the use of two solenoids. At the ANU, an alternative approach for improving beam purity

\footnotetext{
a e-mail: ian.carter@anu.edu.au

b Permanent address: Nuclear Physics Division, Bhabha Atomic Research Centre, Trombay, Mumbai, India

${ }^{\mathrm{c}}$ Current address: School of Electrical, Electronic and Computer Engineering, The University of Western Australia, Perth, WA 6009, Australia
}

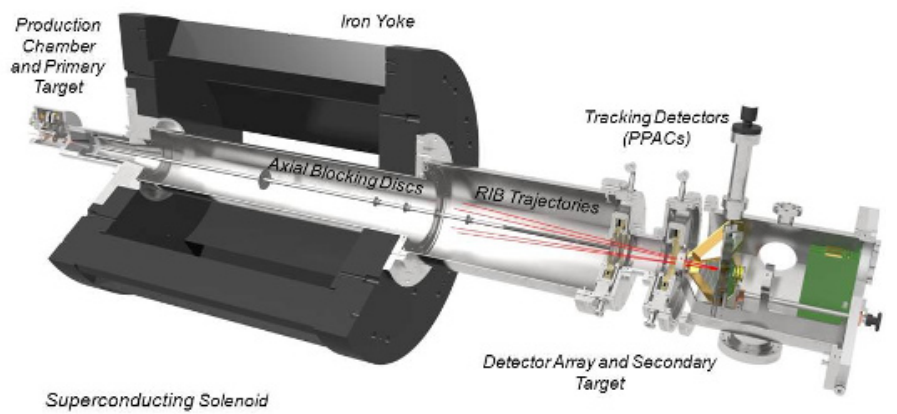

Figure 1. Cross-sectional view of the SOLEROO separator.

is being implemented. To compensate for the lower purity beam from using one solenoid the SOLEROO separator uses a pair of Parallel Plate Avalanche Counters (PPACs) as tracking detectors [3],[7].

RIBs are produced by transfer reactions with the primary target in the production chamber. They enter the solenoid with an angular acceptance of $2^{\circ}-6^{\circ}$. Here they are deflected by the high axial magnetic field and are focused to a secondary target located on the beam axis outside the solenoid. Each ion exiting the solenoid prior to reaching the secondary target will pass through the tracking detector, where it will be electronically identified on an event-by-event basis before hitting the secondary target. This information will allow reconstruction of ion trajec- 


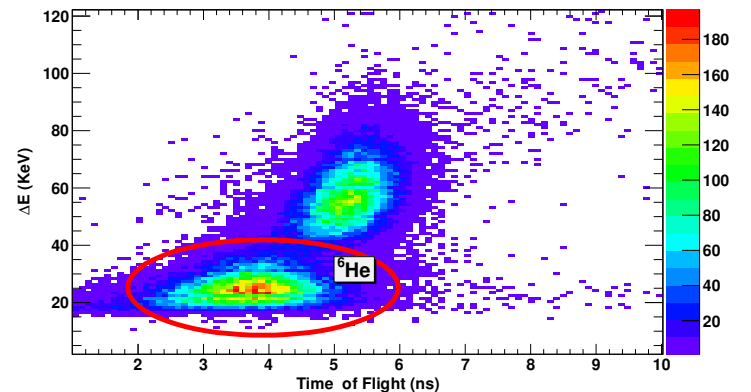

Figure 2. Time of flight of events from PPAC-1 to PPAC-2 spaced $150 \mathrm{~mm}$ apart vs the Energy lost to the gas in PPAC-1. Taken from a measurement using a $30 \mathrm{MeV}{ }^{7} \mathrm{Li}$ beam on a ${ }^{9} \mathrm{Be}$ target at a solenoid field of $3.75 \mathrm{~T}$.

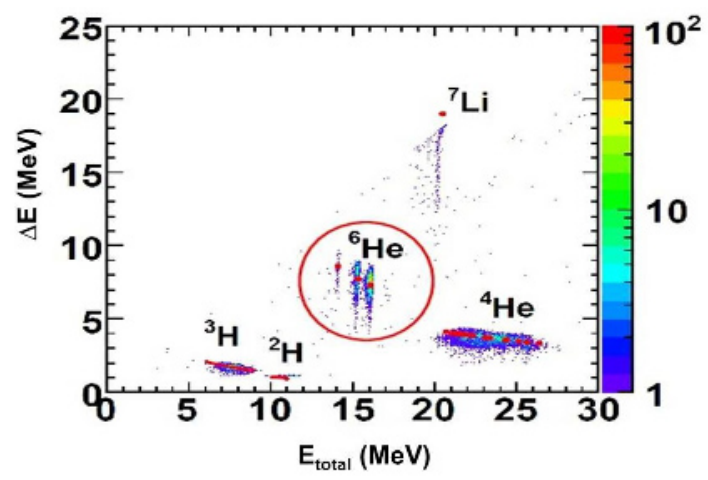

Figure 3. Typical reaction products produced from low-energy reactions of ${ }^{7} \mathrm{Li}+{ }^{9} \mathrm{Be}[4]$ using a $\Delta \mathrm{E}$-E telescope. The tracking detectors will be able to electronically remove the large flux of contaminant species and isolate the RIBs of interest such as ${ }^{6} \mathrm{He}$.

tories which will aid in rejection of contaminant species. Together with time of flight information $\left(\propto \frac{m}{E}\right)$ for the ions passing through one tracking detector to the other, and the energy lost by these ions $\left(\propto \frac{m Z^{2}}{E}\right)$ will allow for separation between ion species arriving at the reaction target (shown in Figure 2). In this figure separation between two distinct groups can be seen. One from predominantly ${ }^{6} \mathrm{He}$, the other from the elasticity scattered ${ }^{7} \mathrm{Li}$ particles which lose more energy in the gas. This separation technique will permit the practical use of RIBs with physical impurities as low as $10 \%$. Figure 3 illustrates typical reaction products detected at the position of the secondary target. The tracking detector will be able to electronically reject the large flux of these contaminant species and select the RIBs of interest. Combined with a large solid angle 512 pixel Si detector array, the system will allow the measurement of cross sections of interest to both nuclear reaction studies and astrophysics.

\section{Tracking Detectors}

The tracking detectors consist of a pair of PPACs [3][7][8] [9][10]. A PPAC is a gas detector that operates in the proportional gas-amplification region. The detectors are each composed of three metallized foils that are mounted parallel to each other with a spacing of $4 \mathrm{~mm}$ between each foil and a square active area of $90 \times 90 \mathrm{~mm}^{2}$. An ionising gas is circulated at 10 Torr of pressure between the foils. The gas used is $\mathrm{C}_{3} \mathrm{~F}_{8}$ with an operating positive (anode) bias of $+820 \mathrm{~V}$ and negative (cathode) bias of $-100 \mathrm{~V}$. This high potential is maintained across the gap between the foils. Incident particles passing through the detector produce free electron-ion pairs. The number of which depends on the density, pressure, depth and ionization potential of the gas, as well as the charge state and energy of the incident particle. The ions accelerate to the foils with negative bias (cathode) and the electrons towards the positively biased foil (anode). The pulse height of the signals measured at this bias from the pre-amplifiers were $50 \mathrm{mV}$ from the anode foil and approximately $10 \mathrm{mV}$ from the cathode foil with rise times in the order of $6 \mathrm{~ns}$. The signal to noise is not an issue for the signals from the anode which are well above noise level. However it becomes problematic for the signals coming from cathode foils which don't have large pulse height above a $5 \mathrm{mV}$ noise level. This issue was addressed by sending the signals through a $45 \mathrm{MHz}$ low-pass noise filter which removed most of the noise.

Since position information is required for tracking, the PPACs used in SOLEROO have two negatively biased foils which are metallized but segmented into parallel strips and are rotated 90 degrees from each other (X and $\mathrm{Y})$ as shown in Figure 4. The position signals in $\mathrm{X}$ or $\mathrm{Y}$ are obtained by connecting each consecutive segmented strip to one tap of a passive delay line [8]. This introduces a time lag for the events which hit a particular strip, and thus introducing a position dependence in the time of the signal collected at either end of the delay line. The electrons produced in the gas feel the positive bias from a central foil (anode) between the $\mathrm{X}$ and $\mathrm{Y}$ foils and the charge collected gives the energy lost by the ions passing through the PPAC. An energy resolution of $40 \%$ from a 5.5 $\mathrm{MeV}^{241} \mathrm{Am}$ alpha-particle source was observed. The time of arrival of the ions passing through the PPAC are also recorded from the electron signal due to the swift mobility of the electrons in the gas compared to the heavier mass ions produced. A time resolution of $950 \mathrm{ps}$ was obtained using the ${ }^{241} \mathrm{Am}$ alpha-particle source.

Angular straggling of beam particles off the foils and gas in the PPACs can effect the fundamental resolution of these detectors. SRIM calculations were carried out to test beam straggling effects on the PPACs [9]. From calculation it was found that straggling in the PPACs have a minimum effect on the detectors performance. The capability of PPACs to operate at a high count rate capacity can be problematic [8]. At higher count rates the signal pulse height reduces. If the amplitude of these pulses drops below set thresholds it will cause the efficiency of the detectors to reduce. The efficiency of the PPACs was measured from the reaction of ${ }^{7} \mathrm{Li}$ on ${ }^{9} \mathrm{Be}$ at $30 \mathrm{MeV}$, at a total bias 


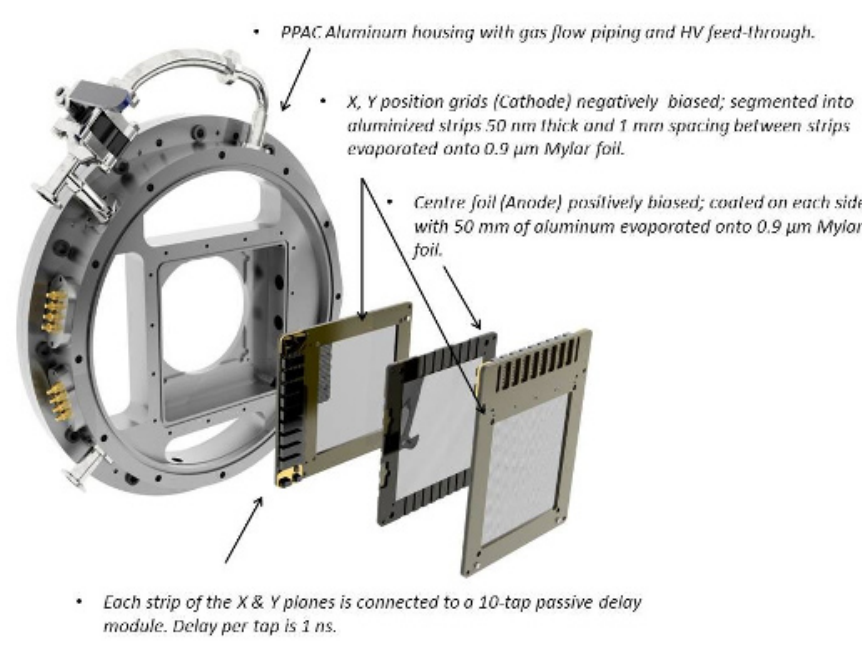

Figure 4. Expanded view of a PPAC assembly.

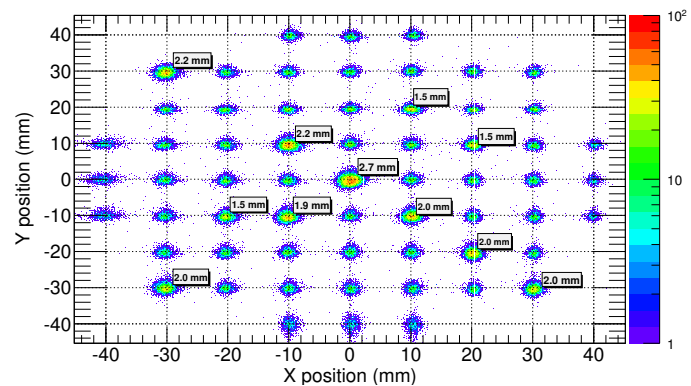

Figure 5. Illustration of the linear response of the detector. The measurement was carried out using a ${ }^{241} \mathrm{Am}$ alpha-particle source mounted facing the PPAC. A mask was designed which was fixed to the entrance of the PPAC window. It had a sequence of fixed sized holes $10 \mathrm{~mm}$ apart from each other in a grid format. The asymmetry arises due to larger size holes been drilled in the mask. This was done in order to identify the specific quadrants of the PPAC. The diameter of the holes are indicated in the figure. Holes not labelled are $1 \mathrm{~mm}$ in diameter.

of $900 \mathrm{~V}$. This was achieved by gating on the ${ }^{6} \mathrm{He}$ group in the $\Delta \mathrm{E}$-E telescope which was placed down-stream in the position of the secondary target. An efficiency of $86 \%$ was recorded for ${ }^{6} \mathrm{He}$ events passing through PPAC- 1 and 2 with a secondary beam rate of $4.4 \times 10^{4}$ counts/s incident of the detectors. At a higher beam rate of $5.5 \times 10^{5}$ counts $/ \mathrm{s}$ the efficiency for this ${ }^{6} \mathrm{He}$ group was $75 \%$. By increasing the bias voltage on the PPACs by $30 \mathrm{~V}$, to a total bias of $930 \mathrm{~V}$, for the lower count rate of $4.4 \times 10^{4}$ counts/s the efficiency increased from $86 \%$ to $99 \%$. For a larger count rate of $1.0 \times 10^{5}$ counts/s the efficiency was $98 \%$ at $930 \mathrm{~V}$.

\section{Tracking Capabilities}

The position sensitivity was examined by designing a mask which was mounted at the entrance of the PPAC window. It had a sequence of fixed sized holes spaced $10 \mathrm{~mm}$ apart from each other. This allowed for a position cali-

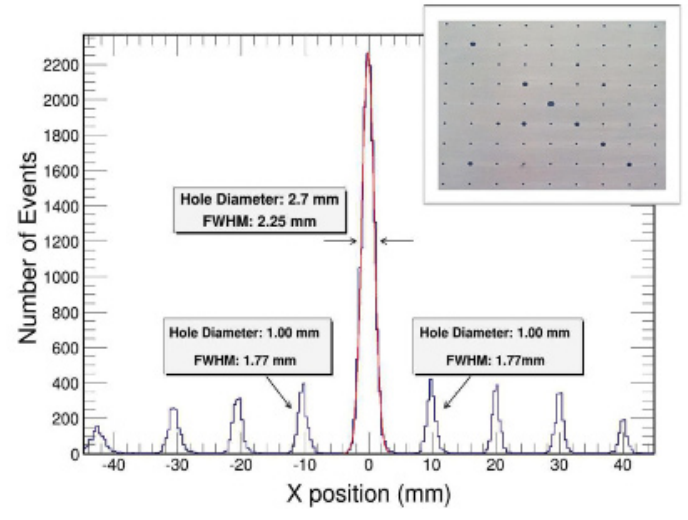

Figure 6. Projection of the $X$ axis gated over events which occurred at $\mathrm{Y}=0 \mathrm{~mm}$ from Figure 5. The insert is an image of the mask used to obtain the position calibration.

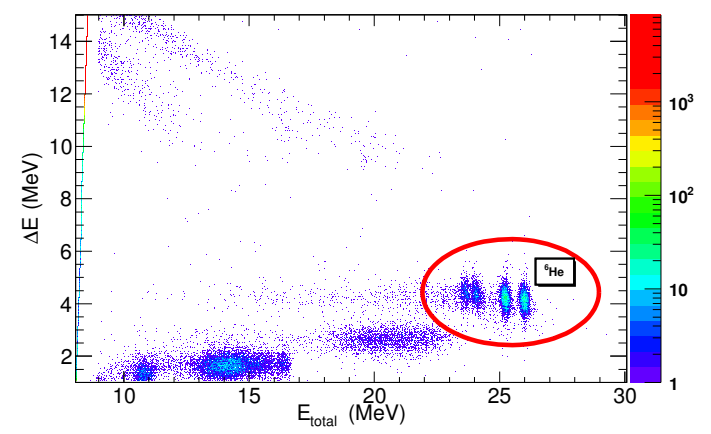

Figure 7. $\Delta \mathrm{E}$ vs $\mathrm{E}$ spectrum from a measurement using a 30 $\mathrm{MeV}^{7} \mathrm{Li}$ beam on a ${ }^{9} \mathrm{Be}$ target, solenoid field of $3.75 \mathrm{~T}$ recorded by the Si telescope placed at the secondary target position beyond the tracking detectors. A gate has been drawn over a ${ }^{6} \mathrm{He}$ group (circled in red). The multi-peaked groups of ${ }^{6} \mathrm{He}$ arise from the excitation of the ${ }^{9} \mathrm{Be}$ target to its first excited states from the primary reaction with the ${ }^{7} \mathrm{Li}$ beam.

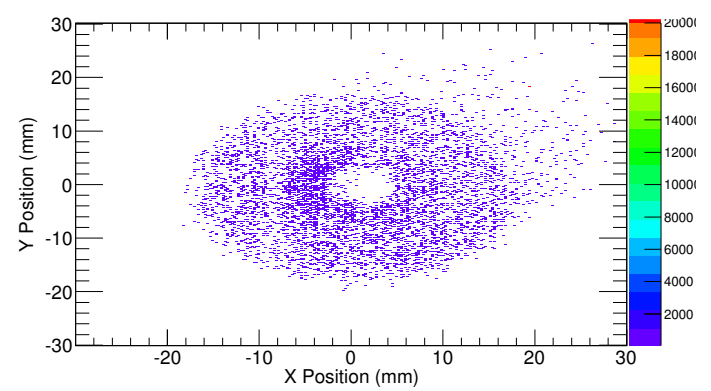

Figure 8. $\mathrm{X}$ and $\mathrm{Y}$ position spectrum from PPAC-1 located at the solenoid exit $150 \mathrm{~mm}$ upstream from PPAC-2. 


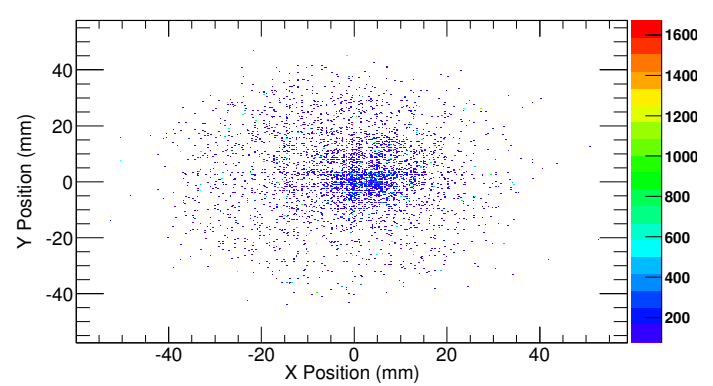

Figure 9. $\mathrm{X}$ and $\mathrm{Y}$ position spectrum from PPAC-2 located 150 $\mathrm{mm}$ downstream from PPAC-1 and $138 \mathrm{~mm}$ upstream from the secondary target position.

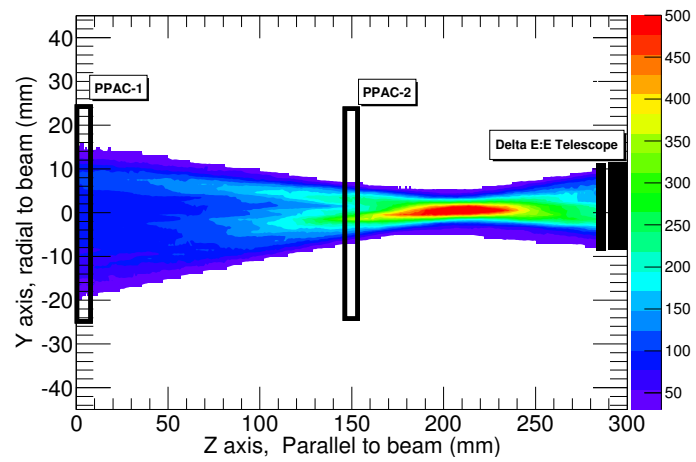

Figure 10. Ion trajectories gated on the reaction group from Figure 7. Trajectories calculated from extrapolating the position information obtained using PPAC- 1 and 2.

bration to be carried out. The results of the measurements obtained using a ${ }^{241} \mathrm{Am}$ alpha-particle source mounted facing the PPAC are shown in Figures 5 and 6. From fitting the peaks with a Gaussian distribution a FWHM of $1.77 \mathrm{~mm}$ was determined from fitting to the events for the $1 \mathrm{~mm}$ diameter apertures.

The position information from the tracking detectors can be used for reconstructing individual ion trajectories and to calculate the position of impact and the angle of incidence at the secondary target position. The tracking capability was checked by making measurements using a $30 \mathrm{MeV}^{7} \mathrm{Li}$ beam on a ${ }^{9} \mathrm{Be}$ target. The products exiting the solenoid were measured by the tracking detector as well as a $\Delta \mathrm{E}-\mathrm{E}$ telescope placed at the secondary target position beyond the tracking detectors (see Figure 1). To illustrate the PPAC tracking capabilities a gate shown in Figure 7 (circled in red) has been drawn over a ${ }^{6} \mathrm{He}$ group in the $\triangle \mathrm{E}$ vs $\mathrm{E}$ spectrum recorded by the $\mathrm{Si}$ telescope. The $\mathrm{X}$ and $\mathrm{Y}$ coordinate position for events inside this gate were obtained, as shown in Figure 8 and 9. Extrapolating from the $\mathrm{X}$ and $\mathrm{Y}$ coordinates from PPAC-1 and 2 allowed a calculation of an interaction point on the $\triangle \mathrm{E}-\mathrm{E}$ telescope. PPAC-2 is located downstream from PPAC-1 by an axial separation of $150 \mathrm{~mm}$ and located $138 \mathrm{~mm}$ upstream from the secondary reaction target. Assuming a straight line trajectory of the ion leaving the solenoid, the reconstruction of the tagged RIBs of interest at the secondary reaction target $\left(X_{R T}, Y_{R T}\right)$ are described by the expressions [3].

$$
\begin{aligned}
& X_{R T}=\frac{138}{150}\left(X_{P P A C-2}-X_{P P A C-1}\right)+X_{P P A C-2} \\
& Y_{R T}=\frac{138}{150}\left(Y_{P P A C-2}-Y_{P P A C-1}\right)+Y_{P P A C-2}
\end{aligned}
$$

An example of reconstructed ion trajectories calculated using Equations 1 and 2 is shown in Figure 10.

\section{Conclusions}

The parallel plate proportional avalanche counter detectors made in-house are in successful operation. We have demonstrated the capabilities of the detectors via in-beam studies. The PPACs are ready to use on-line to track and identify beam species on an event by event basis. On-line testing will involve combining the time of flight between events passing through one PPAC to the other. Combining this information with the energy lost by ions through the PPACs will allow for particle identification and the electronic removal of all unwanted events. This is expected to lead to a dramatic improvement in purity of the RIB species of interest.

Once on-line testing of the PPACs is complete the next step is to integrate our tracking system with a high efficiency 512-pixel wide angle silicon detector array for nuclear reactions studies with radioactive ions [11][12][13].

\section{References}

[1] P.G. Hansen, A.S. Jensen and B. Johnson., Annu. Rev. Nucl. Part. Sci. 45591187 (1995)

[2] J.M. D'Auria and L. Buchmann., Nucl. Instr. and Meth. B 516500 (1991)

[3] R. Rafiei, et al., Nucl. Instr. and Meth., A 63112 (2011)

[4] A.J. Horsley, et al., Nucl., Instr. Meth. in Phys. Res. A 646 (2011) 174

[5] F.D. Becchetti, et al., Nucl. Instr. and Meth. A $\mathbf{5 0 5}$ 377, 2003)

[6] R. Lichtenhaler, et al., Eur. Phys. J. A 2733 (2005)

[7] D.Higginbottom, PPAC Design and Implementation for Exotic Nuclei Research (Thesis unpublished, The Australian National University, 2010)

[8] H. Kumagai, et al., Nucl. Instr. and Meth. A 470 562570 (2001)

[9] R. Rafiei, Ph.D. Thesis, The Australian National University, 2010, published (R. Rafiei, Dynamics of Breakup and Quasifission in Nuclear Reactions, Saarbrucken, Lap (2010)).

[10] S. Ottini-Hustache, et al., Nucl. Instr. and Meth. A 431 3, (1999)

[11] R. Rafiei, et al., Phys. Rev.C 81,024601, (2010)

[12] D. H. Luong, et al., Physics Letters B 695, 105, (2011)

[13] R. Rafiei, et al., Int. J. Mod. Phys. E 20,835, (2011) 